\title{
Field-Tunable One-Sided Higher-Order Topological Hinge States in Dirac Semimetals
}

\author{
Rui Chen, ${ }^{1,2,3}$ Tianyu Liu, ${ }^{4,1}$ C. M. Wang, ${ }^{5,1,2}$ Hai-Zhou Lu, ${ }^{1,2, *}$ and X. C. Xie ${ }^{6,7,8}$ \\ ${ }^{1}$ Shenzhen Institute for Quantum Science and Engineering and Department of Physics, \\ Southern University of Science and Technology (SUSTech), Shenzhen 518055, China \\ ${ }^{2}$ Shenzhen Key Laboratory of Quantum Science and Engineering, Shenzhen 518055, China \\ ${ }^{3}$ School of Physics, Southeast University, Nanjing 211189, China \\ ${ }^{4}$ Max-Planck-Institut für Physik komplexer Systeme, 01187 Dresden, Germany \\ ${ }^{5}$ Department of Physics, Shanghai Normal University, Shanghai 200234, China \\ ${ }^{6}$ International Center for Quantum Materials, School of Physics, Peking University, Beijing 100871, China \\ ${ }^{7}$ Beijing Academy of Quantum Information Sciences, Beijing 100193, China \\ ${ }^{8}$ CAS Center for Excellence in Topological Quantum Computation, \\ University of Chinese Academy of Sciences, Beijing 100190, China
}

\begin{abstract}
Recently, higher-order topological matter and 3D quantum Hall effects have attracted a great amount of attention. The Fermi-arc mechanism of the 3D quantum Hall effect proposed to exist in Weyl semimetals is characterized by the one-sided hinge states, which do not exist in all the previous quantum Hall systems, and more importantly, pose a realistic example of the higher-order topological matter. The experimental effort so far is in the Dirac semimetal $\mathrm{Cd}_{3} \mathrm{As}_{2}$, where however, time-reversal symmetry leads to hinge states on both sides of the top and bottom surfaces, instead of the aspired one-sided hinge states. We propose that under a tilted magnetic field, the hinge states in $\mathrm{Cd}_{3} \mathrm{As}_{2}$-like Dirac semimetals can be one sided, highly tunable by field direction and Fermi energy, and robust against weak disorder. Furthermore, we propose a scanning tunneling Hall measurement to detect the one-sided hinge states. Our results will be insightful for exploring not only the quantum Hall effects beyond two dimensions, but also other higher-order topological insulators in the future.
\end{abstract}

Introduction - Since the discovery of the quantum Hall effect $[1,2]$, tremendous efforts have been devoted to the search for a quantum Hall effect beyond two dimensions [3-30]. Recently, a 3D quantum Hall effect in topological semimetals [31, 32] has attracted a great amount of attention, in which the Fermi arcs from opposite surfaces and the Weyl nodes can form a "Weyl orbit" [33-35] to support the cyclotron motion of electrons driven by magnetic fields and a quantum Hall effect in 3D [Figs. 1(a)1(c)]. More intriguingly, this 3D quantum Hall effect is characterized by the one-sided hinge states, that is, the edge states of the Landau levels lying at one side of the top surface but at the opposite side of the bottom surface [Fig. 1(c)]. The one-sided hinge states do not exist in all the previous quantum Hall systems and can be regarded as an example of the long-sought higher-order topological states of matter [36-74], where a $d$-dimensional system can host $(d-2)$ - or lower-dimensional boundary states protected by both topology and symmetry. The experimental detection of higher-order topological insulators is a challenging problem, because of demanding symmetry requirements. In contrast, the one-sided hinge states in topological semimetals not only may facilitate the detection of the higher-order topological states of matter but also can justify the 3D quantum Hall effect. Nevertheless, the quantum Hall effect has been observed only in slabs of the topological semimetal $\mathrm{Cd}_{3} \mathrm{As}_{2}$, with its mechanism still under debate [75-86]. Moreover, $\mathrm{Cd}_{3} \mathrm{As}_{2}$ is a Dirac semimetal composed of two time-reversed Weyl semimetals, and time-reversal symmetry leads to hinge states on both sides of the top and bottom surfaces [Figs.1(d)-1(e)], instead of the wanted one-sided hinge states [87-90].

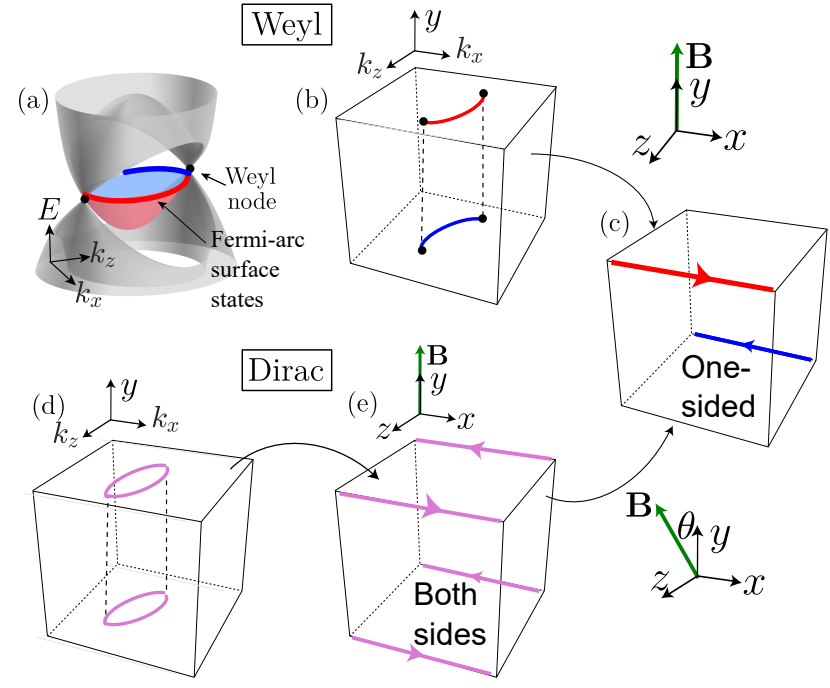

FIG. 1. (a) Energy dispersions of the bulk (gray, $k_{y}=0$ ) and Fermi-arc surface states [red for top and blue for bottom in (b)] of a Weyl semimetal. (b) The Fermi-arc surface states on the top and bottom surfaces can form a $2 \mathrm{D}$ electron gas distributed in the 3D system to support a 3D quantum Hall effect under a magnetic field B. The 3D quantum Hall effect is characterized by the one-sided hinge states (red and blue arrows) in (c), which do not exist in all the previous quantum Hall systems. In the experimentally accessible Dirac semimetals, however, time-reversal symmetry leads to a complete 2D electron gas on each surface $[(d)]$ and hinge states on both sides $[(\mathrm{e})]$, instead of the desired one-sided hinge states. We find that the hinge states in the Dirac semimetal can be one sided, highly tunable by a tilted magnetic field [from (e) to (c)] and Fermi energy [Fig. 2], giving a signature of the Fermi-arc 3D quantum Hall effect and higher-order topological states of matter. There are no hinge states along the $x$ direction because of the assumed translational symmetry. 

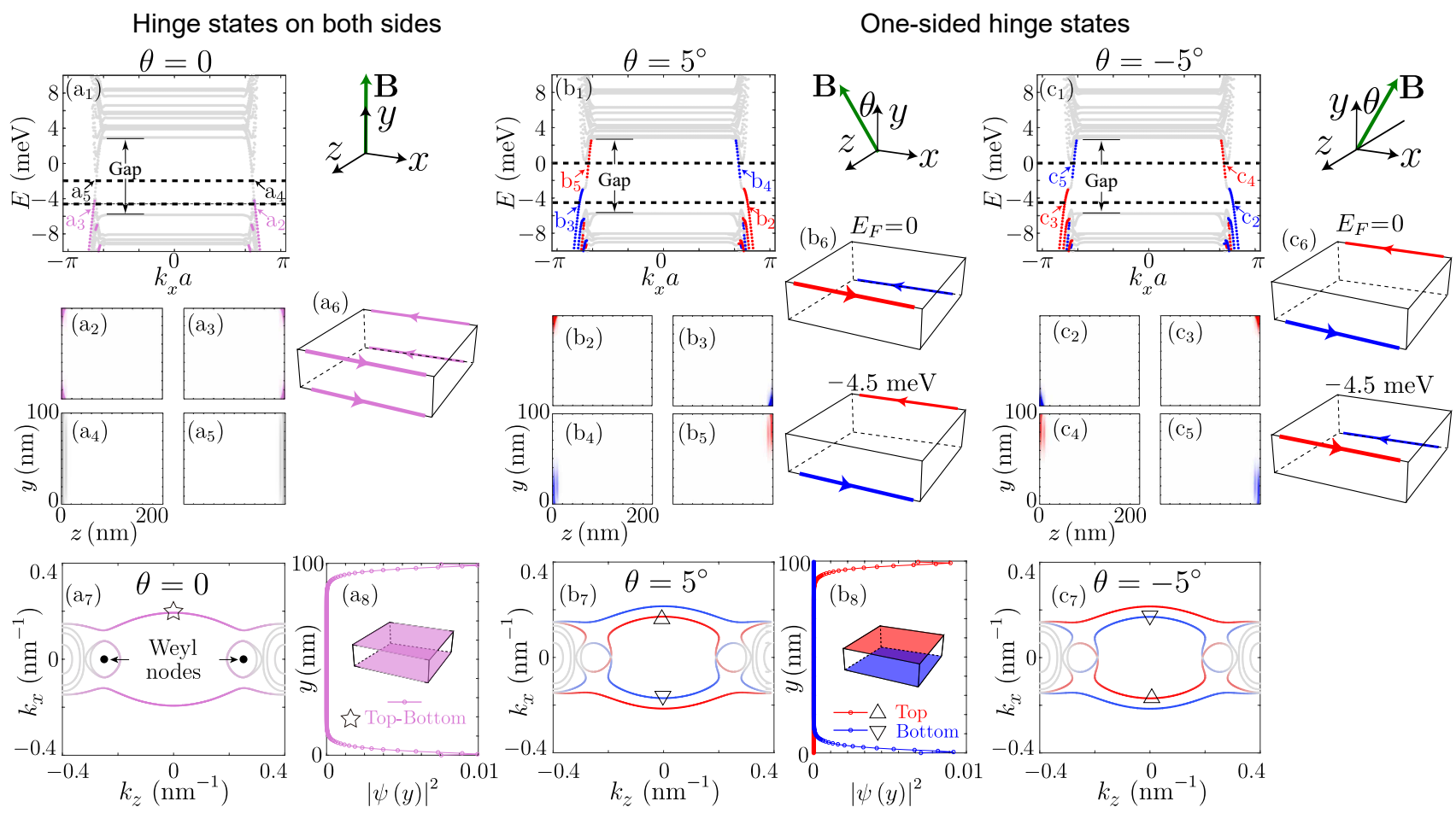

FIG. 2. ( $\left.\mathrm{a}_{1}\right)$ Energy spectrum of the Dirac semimetal ribbon in a magnetic field $B_{y}=15 \mathrm{~T}(\theta=0)$, with open (periodic) boundary conditions along the $y$ and $z(x)$ directions. The ribbon size is $n_{y} a=100 \mathrm{~nm}$ and $n_{z} a=200 \mathrm{~nm}$. The lattice constant $a=1 \mathrm{~nm}$. [( $\left.\left.\mathrm{a}_{2}\right)-\left(\mathrm{a}_{5}\right)\right]$ The wave function distributions for the states marked by the horizontal dash lines in ( $\left.\mathrm{a}_{1}\right)$. Inside the Landau-level gap (at $E_{F}=-4.5 \mathrm{meV}$ ), the hinge states lie on both sides, as schematically illustrated in (a6). (a 7 ) The Fermi surface of the Dirac semimetal slab in $\left(a_{1}\right)$, when considering only the Zeeman effect of the magnetic field. (as) The wave function distribution of the state marked by the star in $\left(a_{7}\right)$. [( $\left.\left.b_{1}\right)-\left(b_{8}\right)\right]$ The same as $\left(a_{1}\right)-\left(a_{8}\right)$, except for $\theta=5^{\circ}$ $\left(B_{z}=B_{y} \tan \theta\right)$. The hinge states become one sided at $E_{F}=0$ and switch their positions at $E_{F}=-4.5 \mathrm{meV}$. [( $\left.\left.\mathrm{c}_{1}\right)-\left(\mathrm{c}_{7}\right)\right] \mathrm{The}$ same as $\left(\mathrm{a}_{1}\right)-\left(\mathrm{a}_{7}\right)$ except for $\theta=-5^{\circ}$. The one-sided hinge states are swapped compared to those in $\left(\mathrm{b}_{1}\right)-\left(\mathrm{b}_{7}\right)$. Red, blue, and purple represent top, bottom, and top-bottom surfaces, respectively.

In this Letter, we propose to realize the one-sided hinge states in $\mathrm{Cd}_{3} \mathrm{As}_{2}$-like Dirac semimetals in a tilted magnetic field [from Figs. 1(e) to 1(c)]. With the help of a realistic model, we numerically calculate the energy spectra and the wave function distributions of $\mathrm{Cd}_{3} \mathrm{As}_{2}$ slabs grown along the experimentally accessible [110] crystallographic direction (Figs. 2 and 3), which show the desired one-sided hinge states, with their positions tunable by the angle of the magnetic field $\theta$ and Fermi energy $E_{F}$. We numerically check the robustness of the one-sided hinge states against weak disorder, and expect the one-sided hinge states to be detectable in the state-of-the-art scanning tunneling Hall measurement (Fig. 4) [91]. This work will not only help identify the Fermi-arc 3D quantum Hall effect but also provide insights for searching for higherorder topological states of matter.

Model - We start with an effective Hamiltonian of the [110]-Dirac semimetal $\mathrm{Cd}_{3} \mathrm{As}_{2}$ slab [87, 92], and consider both the orbital effect (Landau levels) and Zeeman effect of a magnetic field $\mathbf{B}=\left(0, B_{y}, B_{z}=B_{y} \tan \theta\right)$ [18]. Using the Landau gauge, the vector potential of the magnetic field $\mathbf{A}=\left(B_{y} z-B_{z} y, 0,0\right)$, where $\mathbf{B}=\nabla \times \mathbf{A}$. The
Hamiltonian reads

$$
H=\left(\begin{array}{cc}
h_{0}(\mathbf{k})+\Delta_{z} & \Delta_{y} \\
\Delta_{y}^{\dagger} & h_{0}^{*}(-\mathbf{k})-\Delta_{z}
\end{array}\right)
$$

where $h_{0}(\mathbf{k})=\varepsilon_{0}(\mathbf{k}) \sigma_{0}+M(\mathbf{k}) \sigma_{z}+A\left(\bar{k}_{x} C_{\alpha}-k_{y} S_{\alpha}\right) \sigma_{x}-$ $A\left(\bar{k}_{x} S_{\alpha}+k_{y} C_{\alpha}\right) \sigma_{y}$ is for one of the Weyl semimetals with $\varepsilon_{0}(\mathbf{k})=C_{0}+C_{1} k_{z}^{2}+C_{2}\left(\bar{k}_{x}^{2}+k_{y}^{2}\right), M(\mathbf{k})=$ $M_{0}+M_{1} k_{z}^{2}+M_{2}\left(\bar{k}_{x}^{2}+k_{y}^{2}\right) . \bar{k}_{x}=k_{x}-e A_{x} / \hbar, S_{\alpha}=\sin \alpha$, $C_{\alpha}=\cos \alpha$, and $\alpha=-\pi / 4 . \Delta_{y}=-i e^{-i \alpha} B_{y} \mu_{B} G / 4$ and $\Delta_{z}=B_{z} \mu_{B} G / 4$ are the Zeeman energies of the magnetic field. $G=g_{s}\left(\sigma_{0}+\sigma_{z}\right)+g_{p}\left(\sigma_{0}-\sigma_{z}\right), \mu_{B}$ is the Bohr magneton, and $g_{s}=18.6$ and $g_{p}=2$ are the $g$ factors [93]. We adopt the parameters for $\mathrm{Cd}_{3} \mathrm{As}_{2}$ as $C_{0}=-0.0145$ $\mathrm{eV}, C_{1}=10.59 \mathrm{eV} \AA^{2}, C_{2}=11.5 \mathrm{eV} \AA^{2}, M_{0}=0.0205 \mathrm{eV}$, $M_{1}=-18.77 \mathrm{eV} \AA^{2}, M_{2}=-13.5 \mathrm{eV} \AA^{2}$, and $A=0.889$ eVA [94] and discretize the effective Hamiltonian on a 3D simple cubic lattice with a lattice constant $a$ (Supplemental Material [95]). The hinge states can emerge when the magnetic field is perpendicular to direction of the Weyl nodes. For $\mathrm{Cd}_{3} \mathrm{As}_{2}$, the Weyl nodes are along the [001] ( $z$ ) direction, so the slab can be grown along an 
arbitrary superposition of the [100] and [010] ( $x$ and $y)$ directions.

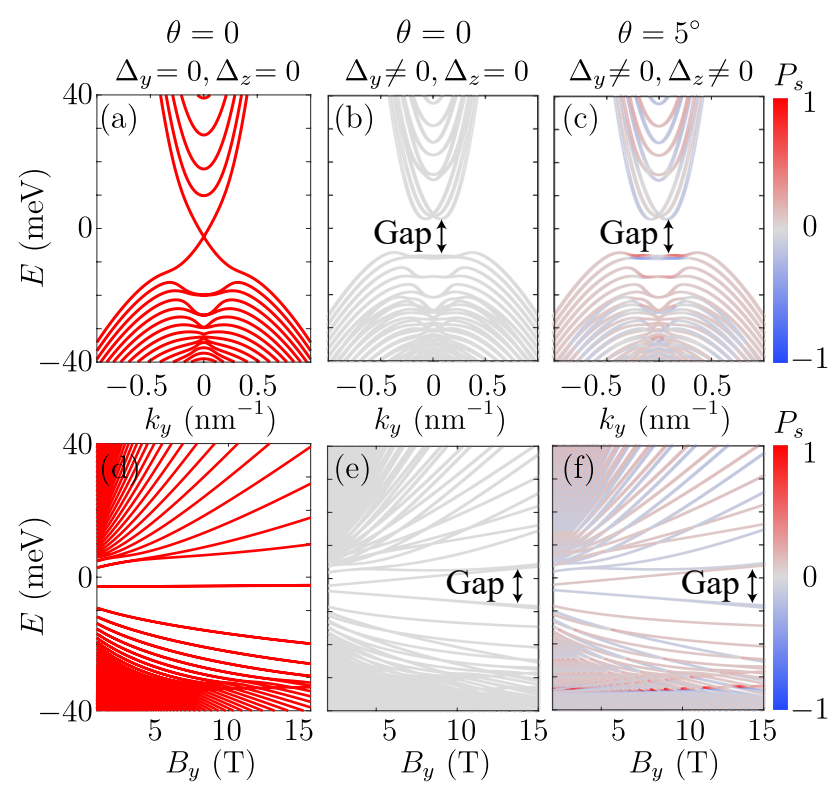

FIG. 3. The Landau bands of the Dirac semimetal (a-c) as functions of the wave vector $k_{y}$ at $B_{y}=15 \mathrm{~T}$ and (d-f) as functions of the magnetic strength $B_{y}$ at $k_{y}=0$. The color bars indicate the weights of $h_{0}(\mathbf{k})$ (red) and $h_{0}^{*}(-\mathbf{k})$ (blue) in Eq. (1). [(a), (d)] $\theta=0$ and the Zeeman coupling $\Delta_{y}=0$. Only the energy spectra for $h_{0}(\mathbf{k})$ are shown as $h_{0}(\mathbf{k})$ and $h_{0}^{*}(-\mathbf{k})$ show the same behavior. [(b), (e)] $\theta=0$ and $\Delta_{y} \neq 0$. $[(\mathrm{c}),(\mathrm{f})] \theta=5^{\circ}$ and the Zeeman energies $\Delta_{y} \neq 0$ and $\Delta_{z} \neq 0$. The orbital effect of $B_{z}$ is not included.

One-sided hinge states in energy spectra and wave function distributions - Figure 2 shows the numerically calculated energy spectra and wave function distributions in magnetic fields for a ribbon of $\mathrm{Cd}_{3} \mathrm{As}_{2}$, with a thickness of $100 \mathrm{~nm}$, width of $200 \mathrm{~nm}$, and periodic boundary condition along the ribbon. $B_{y}$ is fixed at $15 \mathrm{~T}$. Three cases are considered, with the tilting angles $\theta=0$ and $\theta= \pm 5^{\circ}\left(B_{z}=B_{y} \tan \theta\right)$. For each case, the energy spectrum opens a gap near $E=0$. When the magnetic field is applied along the $y$ direction, i.e., $\theta=0$, there appear hinge states [light purple points in Fig. 2(a $\left.a_{1}\right)$ ] on four hinges of the slab, which move along the opposite directions on the opposite side surfaces [Figs. 2( $\left.\mathrm{a}_{2}\right)$, $\left.2\left(\mathrm{a}_{3}\right), 2\left(\mathrm{a}_{6}\right)\right]$. Also, the gray points correspond to the states composed of the Landau levels on the top and bottom surfaces and the chiral states on the side surfaces [Figs. 2(a $\left(a_{4}\right)$ and $2\left(a_{5}\right)$ ]. The side-surface chiral states arise from the quantum Hall effect of the confinementinduced bulk-state subbands.

For the case of a tilted magnetic field $\left(\theta=5^{\circ}\right)$, the hinge states become one sided when the Fermi energy is located in the Landau-level gap [Fig. $\left.2\left(\mathrm{~b}_{1}\right)-2\left(\mathrm{~b}_{6}\right)\right]$. At $E_{F}=-4.5 \mathrm{meV}$, we observe well-localized one-sided hinge states [Fig. $2\left(\mathrm{~b}_{2}\right)-2\left(\mathrm{~b}_{3}\right)$ and $\left.2\left(\mathrm{~b}_{6}\right)\right]$. At $E_{F}=0$ $\mathrm{meV}$, the one-sided hinge states are swapped to the opposite sides compared to those at $E_{F}=-4.5 \mathrm{meV}$ [Fig. $2\left(\mathrm{~b}_{4}\right)-2\left(\mathrm{~b}_{5}\right)$ and $\left.2\left(\mathrm{~b}_{6}\right)\right]$. The $E_{F}=0$ hinge states are not so localized, due to the coupling of the one-sided hinge states to the side-surface chiral states. More interestingly, the one-sided hinge states in Figs. $2\left(b_{1}\right)-2\left(b_{6}\right)$ can be swapped by reversing $\theta$ from positive to negative [Figs. 2(c $\left.\left.\mathrm{c}_{1}\right)-2\left(\mathrm{c}_{6}\right)\right]$.

Hinge states as Landau-level edge states of Fermi arcs - The above evolution of the hinge states can be understood by the Fermi arcs, which are tunable by the Zeeman fields. The Fermi arcs are the topologically protected surface states in the Weyl semimetal. The hinge states are the edge states of the Landau levels of the Fermiarc surface states in magnetic fields. The half-top-halfbottom Fermi-arc surface states in Fig. 1(b) can support the 3D quantum Hall effect and the related one-sided hinge states. However, in only $B_{y}(\theta=0)$, the Zeeman coupling $\Delta_{y}$ couples the Weyl semimetal and its time reversal in the Dirac semimetal, and as a result, the Fermi arcs are evenly distributed on top and bottom surfaces, as shown in Figs. $2\left(a_{7}\right)$ and $\left(a_{8}\right)$. This gives the hinge states on both sides in Fig. $2\left(\mathrm{a}_{6}\right)$. By contrast, the Zeeman splitting $\Delta_{z}$ along the $z$ direction can decouple the Fermi arcs on opposite surfaces [see Figs. $2\left(b_{7}\right)-2\left(b_{8}\right)$ for $\theta=5^{\circ}$ and Fig. $2\left(\mathrm{c}_{7}\right)$ for $\left.\theta=-5^{\circ}\right]$, to yield the one-sided hinge states for $\theta= \pm 5^{\circ}$ (see Supplemental Material [95], Sec. SI for more details).

Landau-level gap that protects one-sided hinge states Our calculations reveal that the Zeeman coupling has to be large enough $\left(B_{y}>10 \mathrm{~T}\right.$ and $\left.\theta>5^{\circ}\right)$ to induce a Landau-level gap large enough to protect the one-sided hinge states (more details in Supplemental Material [95], Sec. SII). To explain the Landau level gaps in Fig. 2, we numerically calculate the energies of the Landau bands of the Dirac semimetal in magnetic fields (Supplemental Material [95]). The external magnetic field has three effects, the Zeeman coupling $\left(\Delta_{y}\right)$ and splitting $\left(\Delta_{z}\right)$ effects and the orbital (Landau levels) effect [Eq. (1)]. First, we consider that there are no Zeeman effects, i.e., $\Delta_{y}=\Delta_{z}=0$. As shown by the energy spectra in Figs. 3(a) and (d), the Landau bands are doubly degenerate because the Dirac semimetal consists of two Weyl semimetals (see Supplemental Material [95], Sec. SI for more details). Second, as shown in Figs. 3(b) and 3(e), a nonzero Zeeman coupling $\Delta_{y} \neq 0$ couples the two Weyl semimetals in Eq. (1) and opens the gap. The size of the gap $E_{g}$ increases linearly with the magnitude of the magnetic field [Fig. 3(e)]. Finally, the Zeeman splitting $\Delta_{z} \neq 0$ in the tilted magnetic field lifts the twofold degeneracy of the energy spectra, and then the one-sided hinge states could appear in the Landau-level gap.

Stability of one-sided hinge states against disorder Now we check the stability of the one-sided hinge states against disorder, by calculating the conductance using the Landauer-Büttiker formula [96-98] and the recursive Green's function method [99, 100] (Supplemental Mate- 

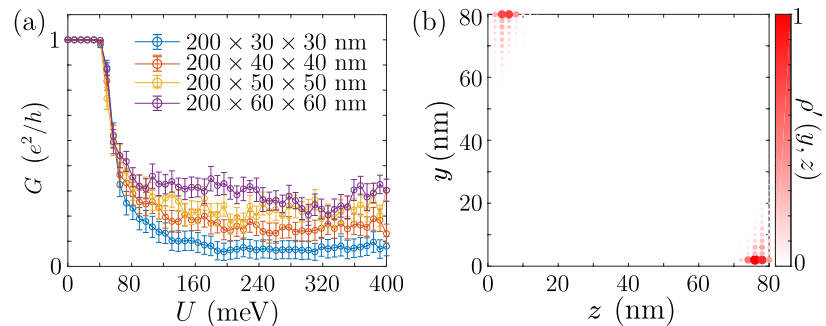

(c)
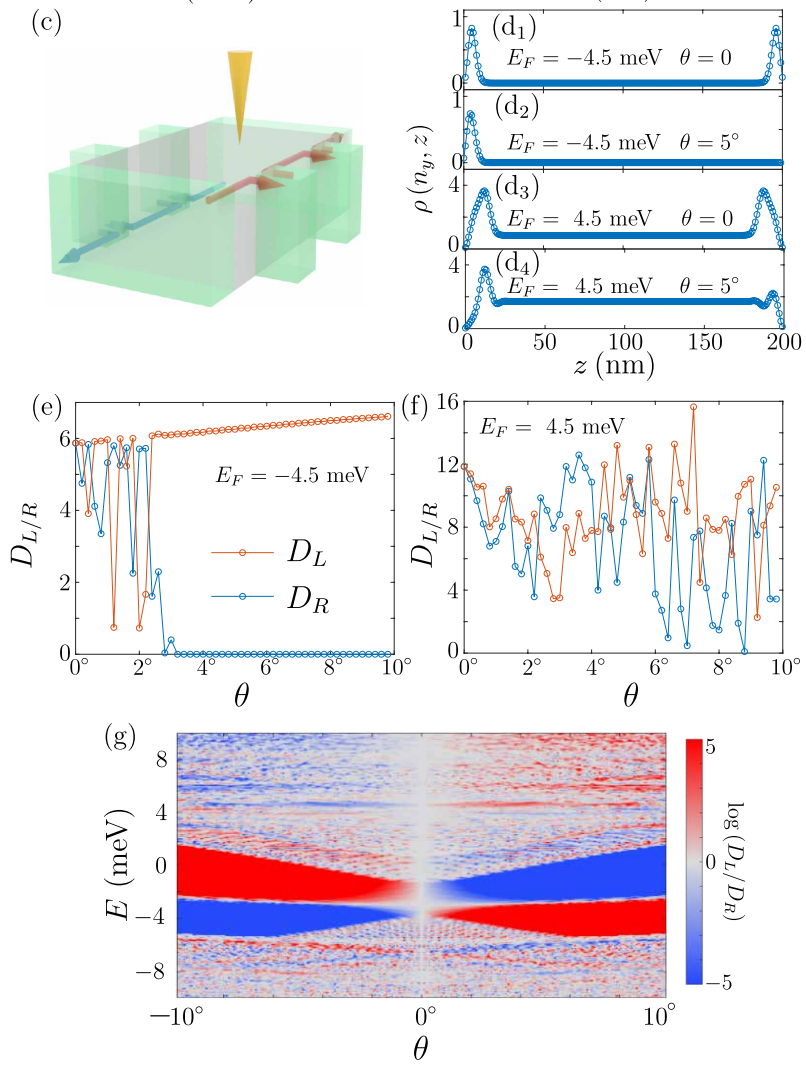

FIG. 4. (a) The disorder-averaged conductance as a function of the disorder strength $U$ for different system sizes. The error bars show the standard deviation for 200 samples. $E_{F}=-4.5$ meV. (b) The disorder-averaged local density of states with system size $n_{x} a=200 \mathrm{~nm}, n_{y} a=n_{z} a=80 \mathrm{~nm} . U=20 \mathrm{meV}$ and $E_{F}=-4.5 \mathrm{meV}$. (c) Schematic of the scanning tunneling Hall measurements for probing the hinge states. Gray, green, and yellow correspond to the Dirac semimetal, in-plane Hall-bar electrodes, and scanning tunneling microscopy tip, respectively. $\left[\left(\mathrm{d}_{1}\right),\left(\mathrm{d}_{2}\right)\right]$ The local density of states $\rho\left(n_{y}, z\right)$ as a function of $z$ at $E_{F}=-4.5 \mathrm{meV}$ for $\theta=0$ and $\theta=5^{\circ}$, respectively. (e) The total local density of states for the 20 outermost layers near the left $(\mathrm{L})$ and right $(\mathrm{R})$ hinges on the topmost layer $D_{L / R}$ as functions of the tilting angle $\theta$ with $E_{F}=-4.5 \mathrm{meV}$. [ $\left.\left(\mathrm{d}_{3}\right),\left(\mathrm{d}_{4}\right),(\mathrm{f})\right]$ The same as $\left(\mathrm{d}_{1}\right),\left(\mathrm{d}_{2}\right)$, and (e), except for $E_{F}=4.5 \mathrm{meV}$. (g) $\log \left(D_{L} / D_{R}\right)$ as a function of $E_{F}$ and $\theta$. In (c)-(g), the ribbon size is $n_{y} a=100 \mathrm{~nm}$ and $n_{z} a=200 \mathrm{~nm}$; the lattice constant $a=1 \mathrm{~nm}$.

rial [95], Sec. SIII). We adopt the Anderson-type disorder by considering random on-site energies fluctuating in the energy interval $[-U, U]$, where $U$ is the disorder strength. Figure 4(a) shows the conductance as a function of the disorder strength $U$ for $E_{F}=-4.5$ $\mathrm{meV}$ and $\theta=5^{\circ}$. In the clean limit, the system is a quantum Hall insulator with a quantized conductance $G=e^{2} / h$. The quantized conductance is from the one-sided hinge states, as confirmed by Fig. 4(b), which shows the disorder-averaged local density of states (Supplemental Material [95], Sec. SIII). With increasing disorder strength, the conductance remains quantized until the disorder strength $U$ exceeds $40 \mathrm{meV}$, which is much larger compared to the Landau-level gap (about $10 \mathrm{meV}$ ). Therefore, we show that the one-sided hinge states are robust against weak disorder. With a further increase in the disorder strength, the conductance drops, but with nonvanishing fluctuations, indicating a signature of the diffusive metal phase $[101,102]$. The diffusive metal phase is generally observed in 3D disordered systems, which further confirms the 3D nature of the Dirac semimetal ribbon that supports the 1D one-sided hinge states. Moreover, the quantized conductance is characterized by a nonzero Chern number $C=1$ (see Supplemental Material [95], Sec. SII for more details).

Scanning tunneling Hall measurement of one-sided hinge states - The one-sided hinge states provide a platform for experimental observation of the Fermiarc 3D quantum Hall effect and higher-order topological states of matter. Naively thinking, the one-sided hinge states can be probed with the help of the scanning tunneling Hall measurements shown in Fig. 4(c), which combines the capabilities of the scanning tunneling microscopy (STM) and the in-plane magnetotransport [91]. The STM tip can probe the local density of states $\rho(E, y, z)=\int_{k_{x}} \sum_{n}\left|\psi_{n}\left(k_{x}, y, z\right)\right|^{2} \delta\left(E-E_{n}\right)$ of the hinge states. Figure $4(\mathrm{~d})$ shows the local density of states of the topmost layer, i.e., $\rho\left(y=n_{y}, z\right)$, as a function of $z$. For $\theta=0$, the two hinges of the topmost layer have the same spectral weight due to the degeneracy of the two Weyl semimetals when $\Delta_{z}=0$. In Fig. $4\left(\mathrm{~d}_{3}\right)$, the nonvanishing local density of states in the central region is attributed to the Landau levels, which contribute to neither the in-plane transport nor the local density of states at the two hinges. Interestingly, for $\theta=5^{\circ}$, the local density of states becomes zero on one side when $E_{F}=-4.5 \mathrm{meV}$ [Fig. $\left.4\left(\mathrm{~d}_{2}\right)\right]$, and is nonzero on both sides when $E_{F}=4.5 \mathrm{meV}$ [Fig. $4\left(\mathrm{~d}_{4}\right)$ ].

Considering the hinge states penetrate up to $20 \mathrm{~nm}$ in Fig. 4(d), we define $D_{L}=\sum_{z=1}^{20} \rho\left(n_{y}, z\right)$ and $D_{R}=$ $\sum_{z=181}^{200} \rho\left(n_{y}, z\right)$ as the total local density of states for the outermost 20 layers near the two hinges. As shown in Figs. 4(e)-4(f), for $E_{F}=-4.5 \mathrm{meV}, D_{R}$ vanishes when $\theta>3^{\circ}$, due to the decoupling of $h(\mathbf{k})$ and $h^{*}(-\mathbf{k})$. Such a behavior is absent for $E_{F}=4.5 \mathrm{meV}$, at which both $D_{L}$ and $D_{R}$ oscillate, due to the band crossings in the energy spectra. The above behaviors can be seen more clearly in the phase diagram shown in Fig. 4(g). The red and blue patches indicate the phase of the one-sided 
hinge states, and they are swapped as $E_{F}$ and $\theta$ changes, corresponding to from Figs. $2\left(\mathrm{~b}_{6}\right)$ and $2\left(\mathrm{c}_{6}\right)$.

Furthermore, we expect that the one-sided hinge states can also be probed by using the Aharonov-Bohm effect, e.g., in a Fabry-Pérot interferometer [103].

This work was supported by the National Natural Science Foundation of China (Grants No. 11925402 and No. 11974249), the National Basic Research Program of China (Grant No. 2015CB921102), the Strategic Priority Research Program of Chinese Academy of Sciences (Grant No. XDB28000000), the Natural Science Foundation of Shanghai (Grant No. 19ZR1437300), Guangdong province (Grants No. 2020KCXTD001 and No. 2016ZT06D348), Shenzhen High-level Special Fund (Grants No. G02206304 and No. G02206404), and the Science, Technology and Innovation Commission of Shenzhen Municipality (Grants No. ZDSYS20170303165926217, No. JCYJ20170412152620376, and No. KYTDPT20181011104202253). R. C. acknowledges support from the project funded by the China Postdoctoral Science Foundation (Grant No. 2019M661678) and the SUSTech Presidential Postdoctoral Fellowship. The numerical calculations were supported by Center for Computational Science and Engineering of SUSTech.

* Corresponding author: luhz@sustech.edu.cn

[1] K. v. Klitzing, G. Dorda, and M. Pepper, "New method for high-accuracy determination of the fine-structure constant based on quantized Hall resistance", Phys. Rev. Lett. 45, 494 (1980).

[2] D. J. Thouless, M. Kohmoto, M. P. Nightingale, and M. den Nijs, "Quantized Hall conductance in a twodimensional periodic potential", Phys. Rev. Lett. 49, 405 (1982).

[3] B. I. Halperin, "Possible states for a three-dimensional electron gas in a strong magnetic field", Jpn. J. Appl. Phys. 26, 1913 (1987).

[4] G. Montambaux and M. Kohmoto, "Quantized Hall effect in three dimensions", Phys. Rev. B 41, 11417 (1990).

[5] M. Kohmoto, B. I. Halperin, and Y.-S. Wu, "Diophantine equation for the three-dimensional quantum Hall effect", Phys. Rev. B 45, 13488 (1992).

[6] M. Koshino, H. Aoki, K. Kuroki, S. Kagoshima, and T. Osada, "Hofstadter butterfly and integer quantum Hall effect in three dimensions", Phys. Rev. Lett. 86, 1062 (2001).

[7] B. A. Bernevig, T. L. Hughes, S. Raghu, and D. P. Arovas, "Theory of the three-dimensional quantum Hall effect in graphite", Phys. Rev. Lett. 99, 146804 (2007).

[8] H. L. Störmer, J. P. Eisenstein, A. C. Gossard, W. Wiegmann, and K. Baldwin, "Quantization of the Hall effect in an anisotropic three-dimensional electronic system", Phys. Rev. Lett. 56, 85 (1986).

[9] J. R. Cooper, W. Kang, P. Auban, G. Montambaux, D. Jérome, and K. Bechgaard, "Quantized Hall effect and a new field-induced phase transition in the organic superconductor (TMTSF) ${ }_{2} \mathrm{PF}_{6}$ ", Phys. Rev. Lett. 63, 1984 (1989).

[10] S. T. Hannahs, J. S. Brooks, W. Kang, L. Y. Chiang, and P. M. Chaikin, "Quantum Hall effect in a bulk crystal", Phys. Rev. Lett. 63, 1988 (1989).

[11] S. Hill, S. Uji, M. Takashita, C. Terakura, T. Terashima, H. Aoki, J. S. Brooks, Z. Fisk, and J. Sarrao, "Bulk quantum Hall effect in $\eta-\mathrm{Mo}_{4} \mathrm{O}_{11} "$, Phys. Rev. B 58, 10778 (1998).

[12] H. Cao, J. Tian, I. Miotkowski, T. Shen, J. Hu, S. Qiao, and Y. P. Chen, "Quantized Hall effect and Shubnikovde Haas oscillations in highly doped $\mathrm{Bi}_{2} \mathrm{Se}_{3}$ : Evidence for layered transport of bulk carriers", Phys. Rev. Lett. 108, 216803 (2012).

[13] H. Masuda, H. Sakai, M. Tokunaga, Y. Yamasaki, A. Miyake, J. Shiogai, et al., "Quantum Hall effect in a bulk antiferromagnet $\mathrm{EuMnBi}_{2}$ with magnetically confined two-dimensional Dirac fermions", Sci. Adv. 2, e1501117 (2016).

[14] Y. Liu, X. Yuan, C. Zhang, Z. Jin, A. Narayan, C. Luo, et al., "Zeeman splitting and dynamical mass generation in Dirac semimetal ZrTes", Nat. Commun. 7, 12516 (2016).

[15] J. Y. Liu, J. Yu, J. L. Ning, H. M. Yi, L. Miao, L. J. Min, et al., "Spin-valley locking and bulk quantum Hall effect in a noncentrosymmetric Dirac semimetal BaMnSb $\mathrm{B}_{2}$ ", Nat. Commun. 12, 4062 (2021).

[16] F. Tang, Y. Ren, P. Wang, R. Zhong, J. Schneeloch, S. A. Yang, et al., "Three-dimensional quantum Hall effect and metal-insulator transition in $\mathrm{ZrTe}_{5}$ ", Nature 569, 537 (2019).

[17] F. Qin, S. Li, Z. Z. Du, C. M. Wang, W. Zhang, D. Yu, H.-Z. Lu, and X. C. Xie, "Theory for the chargedensity-wave mechanism of 3D quantum Hall effect", Phys. Rev. Lett. 125, 206601 (2020).

[18] H. Li, H. Liu, H. Jiang, and X. C. Xie, "3D quantum Hall effect and a global picture of edge states in Weyl semimetals", Phys. Rev. Lett. 125, 036602 (2020).

[19] S.-G. Cheng, H. Jiang, Q.-F. Sun, and X. C. Xie, "Quantum Hall effect in wedge-shaped samples", Phys. Rev. B 102, 075304 (2020).

[20] P. Wang, Y. Ren, F. Tang, P. Wang, T. Hou, H. Zeng, L. Zhang, and Z. Qiao, "Approaching three-dimensional quantum Hall effect in bulk HfTe 5 ", Phys. Rev. B 101, 161201(R) (2020).

[21] R. Ma, D. N. Sheng, and L. Sheng, "Three-dimensional quantum Hall effect and magnetothermoelectric properties in Weyl semimetals", arXiv:2008.13431.

[22] S.-C. Zhang, "A Four-Dimensional Generalization of the Quantum Hall Effect", Science 294, 823 (2001).

[23] M. Lohse, C. Schweizer, H. M. Price, O. Zilberberg, and I. Bloch, "Exploring 4D quantum Hall physics with a 2D topological charge pump", Nature 553, 55 (2018).

[24] O. Zilberberg, S. Huang, J. Guglielmon, M. Wang, K. P. Chen, Y. E. Kraus, and M. C. Rechtsman, "Photonic topological boundary pumping as a probe of $4 \mathrm{D}$ quantum Hall physics", Nature 553, 59 (2018).

[25] H. M. Price, O. Zilberberg, T. Ozawa, I. Carusotto, and N. Goldman, "Four-Dimensional Quantum Hall Effect with Ultracold Atoms", Phys. Rev. Lett. 115, 195303 (2015).

[26] Y. J. Jin, R. Wang, B. W. Xia, B. B. Zheng, and H. Xu, "Three-dimensional quantum anomalous Hall effect in 
ferromagnetic insulators", Phys. Rev. B 98, 081101(R) (2018).

[27] R. A. Molina and J. González, "Surface and 3D Quantum Hall Effects from Engineering of Exceptional Points in Nodal-Line Semimetals", Phys. Rev. Lett. 120, 146601 (2018).

[28] E. Benito-Matías, R. A. Molina, and J. González, "Surface and bulk Landau levels in thin films of Weyl semimetals", Phys. Rev. B 101, 085420 (2020).

[29] M. Chang and L. Sheng, "Three-dimensional quantum Hall effect in the excitonic phase of a Weyl semimetal", Phys. Rev. B 103, 245409 (2021).

[30] M. Chang, H. Geng, L. Sheng, and D. Y. Xing, "Threedimensional quantum Hall effect in Weyl semimetals", Phys. Rev. B 103, 245434 (2021).

[31] C. M. Wang, H.-P. Sun, H.-Z. Lu, and X. C. Xie, "3D quantum Hall effect of Fermi arcs in topological semimetals", Phys. Rev. Lett. 119, 136806 (2017).

[32] H.-Z. Lu, "3D quantum Hall effect", Natl. Sci. Rev. 6, 208 (2018).

[33] A. C. Potter, I. Kimchi, and A. Vishwanath, "Quantum oscillations from surface Fermi arcs in Weyl and Dirac semimetals", Nat. Commun. 5, 5161 (2014).

[34] Y. Zhang, D. Bulmash, P. Hosur, A. C. Potter, and A. Vishwanath, "Quantum oscillations from generic surface Fermi arcs and bulk chiral modes in Weyl semimetals", Sci. Rep. 6, 23741 (2016).

[35] P. J. W. Moll, N. L. Nair, T. Helm, A. C. Potter, I. Kimchi, A. Vishwanath, and J. G. Analytis, "Transport evidence for Fermi-arc-mediated chirality transfer in the Dirac semimetal $\mathrm{Cd}_{3} \mathrm{As}_{2}$ ", Nature 535, 266 (2016).

[36] W. A. Benalcazar, B. A. Bernevig, and T. L. Hughes, "Quantized electric multipole insulators", Science 357, 61 (2017).

[37] J. Langbehn, Y. Peng, L. Trifunovic, F. von Oppen, and P. W. Brouwer, "Reflection-symmetric second-order topological insulators and superconductors", Phys. Rev. Lett. 119, 246401 (2017).

[38] W. A. Benalcazar, B. A. Bernevig, and T. L. Hughes, "Electric multipole moments, topological multipole moment pumping, and chiral hinge states in crystalline insulators", Phys. Rev. B 96, 245115 (2017).

[39] Z. Song, Z. Fang, and C. Fang, "(d-2)-dimensional edge states of rotation symmetry protected topological states", Phys. Rev. Lett. 119, 246402 (2017).

[40] M. Ezawa, "Higher-order topological insulators and semimetals on the breathing kagome and pyrochlore lattices", Phys. Rev. Lett. 120, 026801 (2018).

[41] M. Ezawa, "Topological switch between second-order topological insulators and topological crystalline insulators", Phys. Rev. Lett. 121, 116801 (2018).

[42] F. Schindler, Z. Wang, M. G. Vergniory, A. M. Cook, A. Murani, S. Sengupta, et al., "Higher-order topology in bismuth", Nat. Phys. 14, 918 (2018).

[43] M. Serra-Garcia, V. Peri, R. Süsstrunk, O. R. Bilal, T. Larsen, L. G. Villanueva, and S. D. Huber, "Observation of a phononic quadrupole topological insulator", Nature 555, 342 (2018).

[44] C. W. Peterson, W. A. Benalcazar, T. L. Hughes, and G. Bahl, "A quantized microwave quadrupole insulator with topologically protected corner states", Nature 555, 346 (2018).

[45] J. Noh, W. A. Benalcazar, S. Huang, M. J. Collins, K. P. Chen, T. L. Hughes, and M. C. Rechtsman, "Topologi- cal protection of photonic mid-gap defect modes", Nat. Photonics 12, 408 (2018).

[46] S. Imhof, C. Berger, F. Bayer, J. Brehm, L. W. Molenkamp, T. Kiessling, et al., "Topolectrical-circuit realization of topological corner modes", Nat. Phys. 14, 925 (2018).

[47] F. Liu, H.-Y. Deng, and K. Wakabayashi, "Helical topological edge states in a quadrupole phase", Phys. Rev. Lett. 122, 086804 (2019).

[48] D. Călugăru, V. Juričić, and B. Roy, "Higher-order topological phases: A general principle of construction", Phys. Rev. B 99, 041301 (2019).

[49] B. Roy, "Antiunitary symmetry protected higher-order topological phases", Phys. Rev. Research 1, 032048 (2019).

[50] M. Serra-Garcia, R. Süsstrunk, and S. D. Huber, "Observation of quadrupole transitions and edge mode topology in an LC circuit network", Phys. Rev. B 99, 020304(R) (2019).

[51] R. Chen, C.-Z. Chen, J.-H. Gao, B. Zhou, and D.-H. $\mathrm{Xu}$, "Higher-order topological insulators in quasicrystals", Phys. Rev. Lett. 124, 036803 (2020).

[52] C.-B. Hua, R. Chen, B. Zhou, and D.-H. Xu, "Higherorder topological insulator in a dodecagonal quasicrystal", Phys. Rev. B 102, 241102(R) (2020).

[53] Z.-R. Liu, L.-H. Hu, C.-Z. Chen, B. Zhou, and D.-H. Xu, "Topological excitonic corner states and nodal phase in bilayer quantum spin Hall insulators", arXiv:2101.00923 (2021).

[54] R. Queiroz and A. Stern, "Splitting the hinge mode of higher-order topological insulators", Phys. Rev. Lett. 123, 036802 (2019).

[55] M. Sitte, A. Rosch, E. Altman, and L. Fritz, "Topological Insulators in Magnetic Fields: Quantum Hall Effect and Edge Channels with a Nonquantized $\theta$ Term", Phys. Rev. Lett. 108, 126807 (2012).

[56] F. Zhang, C. L. Kane, and E. J. Mele, "Surface state magnetization and chiral edge states on topological insulators", Phys. Rev. Lett. 110, 046404 (2013).

[57] Y. Otaki and T. Fukui, "Higher-order topological insulators in a magnetic field", Phys. Rev. B 100, 245108 (2019).

[58] F. Schindler, A. M. Cook, M. G. Vergniory, Z. Wang, S. S. P. Parkin, B. A. Bernevig, and T. Neupert, "Higher-order topological insulators", Sci. Adv. 4, eaat0346 (2018).

[59] Z. Yan, F. Song, and Z. Wang, "Majorana corner modes in a high-temperature platform", Phys. Rev. Lett. 121, 096803 (2018).

[60] Z. Yan, "Higher-order topological odd-parity superconductors", Phys. Rev. Lett. 123, 177001 (2019).

[61] D. Varjas, A. Lau, K. Pöyhönen, A. R. Akhmerov, D. I. Pikulin, and I. C. Fulga, "Topological phases without crystalline counterparts", Phys. Rev. Lett. 123, 196401 (2019).

[62] Y.-B. Choi, Y. Xie, C.-Z. Chen, J. Park, S.-B. Song, $\mathrm{J}$. Yoon, et al., "Evidence of higher-order topology in multilayer $\mathrm{WTe}_{2}$ from Josephson coupling through anisotropic hinge states", Nat. Mater. 19, 974 (2020).

[63] S. A. A. Ghorashi, T. Li, and T. L. Hughes, "Higherorder Weyl semimetals", Phys. Rev. Lett. 125, 266804 (2020).

[64] H.-X. Wang, Z.-K. Lin, B. Jiang, G.-Y. Guo, and J.H. Jiang, "Higher-Order Weyl Semimetals", Phys. Rev. 
Lett. 125, 146401 (2020).

[65] B. J. Wieder, Z. Wang, J. Cano, X. Dai, L. M. Schoop, B. Bradlyn, and B. A. Bernevig, "Strong and fragile topological Dirac semimetals with higher-order Fermi arcs", Nat. Commun. 11, 627 (2020).

[66] K. Wang, J.-X. Dai, L. B. Shao, S. A. Yang, and Y. X. Zhao, "Boundary Criticality of $\mathcal{P} \mathcal{T}$-Invariant Topology and Second-Order Nodal-Line Semimetals", Phys. Rev. Lett. 125, 126403 (2020).

[67] S. A. Hassani Gangaraj, C. Valagiannopoulos, and F. Monticone, "Topological scattering resonances at ultralow frequencies", Phys. Rev. Research 2, 023180 (2020).

[68] A. Sarsen and C. Valagiannopoulos, "Robust polarization twist by pairs of multilayers with tilted optical axes", Phys. Rev. B 99, 115304 (2019).

[69] M. Kheirkhah, Y. Nagai, C. Chen, and F. Marsiglio, "Majorana corner flat bands in two-dimensional secondorder topological superconductors", Phys. Rev. B 101, $104502(2020)$.

[70] M. Kheirkhah, Z. Yan, Y. Nagai, and F. Marsiglio, "First- and second-order topological superconductivity and temperature-driven topological phase transitions in the extended Hubbard model with spin-orbit coupling", Phys. Rev. Lett. 125, 017001 (2020).

[71] B. Huang and W. V. Liu, "Floquet Higher-Order Topological Insulators with Anomalous Dynamical Polarization", Phys. Rev. Lett. 124, 216601 (2020).

[72] H. Hu, B. Huang, E. Zhao, and W. V. Liu, "Dynamical Singularities of Floquet Higher-Order Topological Insulators", Phys. Rev. Lett. 124, 057001 (2020).

[73] B. Xie, H.-X. Wang, X. Zhang, P. Zhan, J.-H. Jiang, M. Lu, and Y. Chen, "Higher-order band topology", Nat. Rev. Phys. 3, 520 (2021).

[74] C.-Z. Li, A.-Q. Wang, C. Li, W.-Z. Zheng, A. Brinkman, D.-P. Yu, and Z.-M. Liao, "Reducing Electronic Transport Dimension to Topological Hinge States by Increasing Geometry Size of Dirac Semimetal Josephson Junctions", Phys. Rev. Lett. 124, 156601 (2020).

[75] C. Zhang, A. Narayan, S. Lu, J. Zhang, H. Zhang, Z. Ni, et al., "Evolution of Weyl orbit and quantum Hall effect in Dirac semimetal $\mathrm{Cd}_{3} \mathrm{As}_{2}$ ", Nat. Commun. 8, 1272 (2017).

[76] M. Uchida, Y. Nakazawa, S. Nishihaya, K. Akiba, M. Kriener, Y. Kozuka, et al., "Quantum Hall states observed in thin films of Dirac semimetal $\mathrm{Cd}_{3} \mathrm{As}_{2}$ ", Nat. commun. 8, 2274 (2017).

[77] T. Schumann, L. Galletti, D. A. Kealhofer, H. Kim, M. Goyal, and S. Stemmer, "Observation of the quantum Hall effect in confined films of the three-dimensional Dirac semimetal $\mathrm{Cd}_{3} \mathrm{As}_{2}$ ", Phys. Rev. Lett. 120, 016801 (2018).

[78] L. Galletti, T. Schumann, O. F. Shoron, M. Goyal, D. A. Kealhofer, H. Kim, and S. Stemmer, "Two-dimensional Dirac fermions in thin films of $\mathrm{Cd}_{3} \mathrm{As}_{2}$ ", Phys. Rev. B 97, 115132 (2018).

[79] S. Nishihaya, M. Uchida, Y. Nakazawa, M. Kriener, Y. Kozuka, Y. Taguchi, and M. Kawasaki, "Gatetuned quantum Hall states in Dirac semimetal $\left(\mathrm{Cd}_{1-x} \mathrm{Zn}_{x}\right) \mathrm{Ass}_{3} "$, Sci. Adv. 4, eaar5668 (2018).

[80] M. Goyal, L. Galletti, S. Salmani-Rezaie, T. Schumann, D. A. Kealhofer, and S. Stemmer, "Thickness dependence of the quantum Hall effect in films of the threedimensional Dirac semimetal $\mathrm{Cd}_{3} \mathrm{As}_{2}$ ", APL Materials
6, 026105 (2018).

[81] C. Zhang, Y. Zhang, X. Yuan, S. Lu, J. Zhang, A. Narayan, et al., "Quantum Hall effect based on Weyl orbit in $\mathrm{Cd}_{3} \mathrm{Ass}_{2}$ ", Nature 565, 331 (2019).

[82] S. Wang, B.-C. Lin, W.-Z. Zheng, D. Yu, and Z.M. Liao, "Fano Interference between Bulk and Surface States of a Dirac Semimetal $\mathrm{Cd}_{3} \mathrm{As}_{2}$ Nanowire", Phys. Rev. Lett. 120, 257701 (2018).

[83] S. Nishihaya, M. Uchida, Y. Nakazawa, R. Kurihara, K. Akiba, M. Kriener, A. Miyake, Y. Taguchi, M. Tokunaga, and M. Kawasaki, "Quantized surface transport in topological Dirac semimetal films", Nat. Commun. 10, 2564 (2019).

[84] B.-C. Lin, S. Wang, S. Wiedmann, J.-M. Lu, W.-Z. Zheng, D. Yu, and Z.-M. Liao, "Observation of an OddInteger Quantum Hall Effect from Topological Surface States in $\mathrm{Cd}_{3} \mathrm{Ass}_{2}$ ", Phys. Rev. Lett. 122, 036602 (2019).

[85] H. F. Yang, L. X. Yang, Z. K. Liu, Y. Sun, C. Chen, H. Peng, et al., "Topological Lifshitz transitions and Fermi arc manipulation in Weyl semimetal NbAs", Nat. Commun. 10, 3478 (2019).

[86] D. A. Kealhofer, L. Galletti, T. Schumann, A. Suslov, and S. Stemmer, "Topological Insulator State and Collapse of the Quantum Hall Effect in a ThreeDimensional Dirac Semimetal Heterojunction", Phys. Rev. X 10, 011050 (2020).

[87] Z. Wang, H. Weng, Q. Wu, X. Dai, and Z. Fang, "Three-dimensional Dirac semimetal and quantum transport in $\mathrm{Cd}_{3} \mathrm{As}_{2}$ ", Phys. Rev. B 88, 125427 (2013).

[88] T. Liang, Q. Gibson, M. N. Ali, M. H. Liu, R. J. Cava, and N. P. Ong, "Ultrahigh mobility and giant magnetoresistance in the Dirac semimetal $\mathrm{Cd}_{3} \mathrm{As}_{2}$ ", Nature Mater. 14, 280 (2015).

[89] Z. K. Liu, J. Jiang, B. Zhou, Z. J. Wang, Y. Zhang, H. M. Weng, et al., "A stable three-dimensional topological Dirac semimetal $\mathrm{Cd}_{3} \mathrm{As}_{2}$ ", Nature Mater. 13, 677 (2014).

[90] S. Borisenko, Q. Gibson, D. Evtushinsky, V. Zabolotnyy, B. Büchner, and R. J. Cava, "Experimental realization of a three-dimensional Dirac semimetal", Phys. Rev. Lett. 113, 027603 (2014).

[91] J. Schwenk, S. Kim, J. Berwanger, F. Ghahari, D. Walkup, M. R. Slot, et al., "Achieving $\mu \mathrm{eV}$ tunneling resolution in an in-operando scanning tunneling microscopy, atomic force microscopy, and magnetotransport system for quantum materials research", Rev. Sci. Instrum. 91, 071101 (2020).

[92] L.-X. Wang, S. Wang, J.-G. Li, C.-Z. Li, D. Yu, and Z.-M. Liao, "Universal conductance fluctuations in Dirac semimetal $\mathrm{Cd}_{3} \mathrm{As}_{2}$ nanowires", Phys. Rev. B 94, 161402(R) (2016).

[93] S. Jeon, B. B. Zhou, A. Gyenis, B. E. Feldman, I. Kimchi, A. C. Potter, Q. D. Gibson, R. J. Cava, A. Vishwanath, and A. Yazdani, "Landau quantization and quasiparticle interference in the three-dimensional Dirac semimetal $\mathrm{Cd}_{3} \mathrm{As}_{2}$ ", Nature Mater. 13, 851 (2014).

[94] J. Cano, B. Bradlyn, Z. Wang, M. Hirschberger, N. P. Ong, and B. A. Bernevig, "Chiral anomaly factory: Creating Weyl fermions with a magnetic field", Phys. Rev. B 95, 161306(R) (2017).

[95] See Supplemental Materias for more details of the $\mathrm{k} \cdot \mathrm{p}$ model, tight-binding model, disorder-averaged conductance and local density of states, which includes Refs. [79, 83, 86, 87, 93, 94, 96-100, 104-114]. 
[96] R. Landauer, "Electrical resistance of disordered onedimensional lattices", Philos. Mag. 21, 863 (1970).

[97] M. Büttiker, "Absence of backscattering in the quantum Hall effect in multiprobe conductors", Phys. Rev. B 38, 9375 (1988).

[98] D. S. Fisher and P. A. Lee, "Relation between conductivity and transmission matrix", Phys. Rev. B 23, 6851 (1981).

[99] A. MacKinnon, "The calculation of transport properties and density of states of disordered solids", Z. Phys. B 59, 385 (1985).

[100] G. Metalidis and P. Bruno, "Green's function technique for studying electron flow in two-dimensional mesoscopic samples", Phys. Rev. B 72, 235304 (2005).

[101] C.-Z. Chen, J. Song, H. Jiang, Q.-f. Sun, Z. Wang, and X. C. Xie, "Disorder and Metal-Insulator Transitions in Weyl Semimetals", Phys. Rev. Lett. 115, 246603 (2015).

[102] R. Chen, C.-Z. Chen, J.-H. Sun, B. Zhou, and D.-H. Xu, "Phase diagrams of Weyl semimetals with competing intraorbital and interorbital disorders", Phys. Rev. B 97, 235109 (2018).

[103] C.-A. Li, S.-B. Zhang, J. Li, and B. Trauzettel, "Higher-Order Fabry-Pérot Interferometer from Topological Hinge States", Phys. Rev. Lett. 127, 026803 (2021).

[104] T. Schumann, M. Goyal, H. Kim, and S. Stemmer, "Molecular beam epitaxy of $\mathrm{Cd}_{3} \mathrm{As}_{2}$ on a III-V substrate", APL Materials 4, 126110 (2016).

[105] D. N. Sheng, Z. Y. Weng, L. Sheng, and F. D. M.
Haldane, "Quantum Spin-Hall Effect and Topologically Invariant Chern Numbers", Phys. Rev. Lett. 97, 036808 (2006).

[106] H. Li, L. Sheng, D. N. Sheng, and D. Y. Xing, "Chern number of thin films of the topological insulator $\mathrm{Bi}_{2} \mathrm{Se}_{3}$ ", Phys. Rev. B 82, 165104 (2010).

[107] E. Prodan, "Robustness of the spin-Chern number", Phys. Rev. B 80, 125327 (2009).

[108] R. Bianco and R. Resta, "Mapping topological order in coordinate space", Phys. Rev. B 84, 241106(R) (2011).

[109] A. Marrazzo and R. Resta, "Locality of the anomalous Hall conductivity", Phys. Rev. B 95, 121114(R) (2017).

[110] M. D. Caio, G. Möller, N. R. Cooper, and M. J. Bhaseen, "Topological marker currents in Chern insulators", Nat. Phys. 15, 257 (2019).

[111] O. Pozo, C. Repellin, and A. G. Grushin, "Quantization in chiral higher order topological insulators: Circular dichroism and local Chern marker", Phys. Rev. Lett. 123, 247401 (2019).

[112] D. Varjas, M. Fruchart, A. R. Akhmerov, and P. M. Perez-Piskunow, "Computation of topological phase diagram of disordered $\mathrm{Pb}_{1-x} \mathrm{Sn}_{x}$ Te using the kernel polynomial method", Phys. Rev. Research 2, 013229 (2020).

[113] A. Weiße, G. Wellein, A. Alvermann, and H. Fehske, "The kernel polynomial method", Rev. Mod. Phys. 78, 275 (2006).

[114] S. Datta, Electronic Transport in Mesoscopic Systems (Cambridge University Press, 1997). 